\title{
Detailed investigation of ignition by hot gas jets
}

\author{
R. Sadanandan ${ }^{\mathrm{a}, *}$, D. Markus ${ }^{\mathrm{a}}$, R. Schieß1 ${ }^{\mathrm{b}}$, U. Maas ${ }^{\mathrm{b}}$, \\ J. Olofsson ${ }^{\mathrm{c}}, \mathrm{H}$. Seyfried ${ }^{\mathrm{c}}, \mathrm{M}$. Richter ${ }^{\mathrm{c}}$, M. Aldén ${ }^{\mathrm{c}}$ \\ ${ }^{a}$ Physikalisch Technische Bundesanstalt, Braunschweig, Germany \\ ${ }^{\mathrm{b}}$ Universität Karlsruhe, Institut für Technische Thermodynamik, Germany \\ ${ }^{\mathrm{c}}$ Lund Institute of Technology, Division of Combustion Physics, Sweden
}

\begin{abstract}
Experimental and numerical investigations of the ignition of hydrogen/air mixtures by jets of hot exhaust gases are reported. An experimental realisation of such an ignition process, where a jet of hot exhaust gas impinges through a narrow nozzle into a quiescent hydrogen/air mixture, possibly initiating ignition and combustion, is studied. High-speed laser-induced fluorescence (LIF) image sequences of the hydroxyl radical $(\mathrm{OH})$ and laser Schlieren methods are used to gain information about the spatial and temporal evolution of the ignition process. Recording temporally resolved pressure traces yields information about ambient conditions for the process. Numerical experiments are performed that allow linking these observables to certain characteristic states of the gas mixture. The outcome of numerical modelling and experiments indicates the important influence of the hot jet temperature and speed of mixing between the hot and cold gases on the ignition process. The results show the quenching of the flame inside the nozzle and the subsequent ignition of the mixture by the hot exhaust jet. These detailed examinations of the ignition process improve the knowledge concerning flame transmission out of electrical equipment of the type of protection flameproof enclosure.
\end{abstract}

(C) 2006 The Combustion Institute. Published by Elsevier Inc. All rights reserved.

Keywords: Jet ignition; OH LIF; High-speed laser diagnostics

\section{Introduction and theoretical background}

Ignition of fuel/air mixtures by impinging hot gas jets is a very important process in various fields ranging from industrial explosions and nuclear safety to supersonic combustors and to the development of combustion engines [1]. In explosive environments, hot jet ignition may take

\footnotetext{
* Corresponding author. Present address: German Aerospace Center (DLR), Institute of Combustion Technology, Pfaffenwaldring 38-40, D-70569 Stuttgart, Germany. Fax: +49 7116862578.

E-mail address: Rajesh.Sadanandan@dlr.de
} (R. Sadanandan). place due to a hot jet escaping from flameproof enclosures through inevitable gaps [2], e.g., like the ones present at the shaft bearing of an electrical motor. Closer analysis shows that hot jet ignition is a complicated process involving turbulent mixing of exhaust and fresh gas coupled with chemical reactions. It becomes even more complex at supersonic flow regimes when gas dynamic effects on the jet temperature can have a profound influence on the ignition process. The influence of pressure, temperature, and chemical kinetics on the ignition process has been examined widely for premixed $\mathrm{H}_{2}$ /air mixtures [3]. The role of strain and turbulence has been studied for nonpremixed $\mathrm{H}_{2} /$ air mixtures experimentally [4] and 
numerically [5]. However, theoretical and experimental investigations on the fundamental mechanisms behind the complex coupling of chemical reactions and turbulence are very sparse for hot jet ignition. Literature on the effects of mixing and chemistry on the ignition initiation by hot turbulent jets can be found in the earlier works done by Meyer et al. [6] and Philips [7]. More recent studies have examined the ignition processes from the view of explosion protection determining critical nozzle diameters for flame transmission $[8,9]$.

The current study focuses on the ignition of $28 \mathrm{~mol} \% / 72 \mathrm{~mol} \%$ hydrogen/air mixtures, which is a standard test gas concentration for gas group IIC $[2,10]$, following the gas grouping for electrical apparatus in IEC 60079-0 [11]. The strategy is to use a combination of experimental observation and numerical simulation to identify the dominating processes. Experiments were performed in an optically accessible, constant volume combustion cell. In this cell, a jet of burned gases impinging into an unburned hydrogen/air mixture at room temperature is created. The hydroxyl radical $(\mathrm{OH})$ is used as a marker of the reaction zone as it is an important intermediate species that is formed during the combustion process [12,13]. The temporal development of the hot jet penetration into the fresh mixture is observed using combined laser Schlieren and OH-LIF [14] visualisation sequenced at high repetition rates. Experiments were performed with different pressure ratios over the nozzle and different nozzle diameters in order to study the influence of different jet velocities on the gas expansion and ignition processes. To aid the interpretation of the measured $\mathrm{OH}-$ LIF signals, simple numerical simulations were carried out yielding detailed information on the mechanisms involved in the ignition process.

\section{Methodology}

The measurement object was a jet of hot exhaust gas impinging into a quiescent hydrogen/air mixture which was generated in a dedicated test cell with optical access. A schematic of the combustion cell is shown in Fig. 1. It consists of two chambers which are interconnected by means of a nozzle. The cylindrical smaller chamber (length $80 \mathrm{~mm}$, diameter $60 \mathrm{~mm}$, volume $0.226 \mathrm{~L}$ ) was flanged to a second chamber (volume $12 \mathrm{~L}$ ) which is equipped with three quartz windows for optical access. In each experiment, both chambers were filled with $28 \mathrm{~mol} \% / 72 \mathrm{~mol} \%$ hydrogen/air mixtures up to atmospheric pressure. The $\mathrm{H}_{2} /$ air mixture in the first chamber was then ignited by means of a spark plug located at an ignition distance $X_{\mathrm{i}}$, defined as the distance between the spark plug centre and the nozzle. This

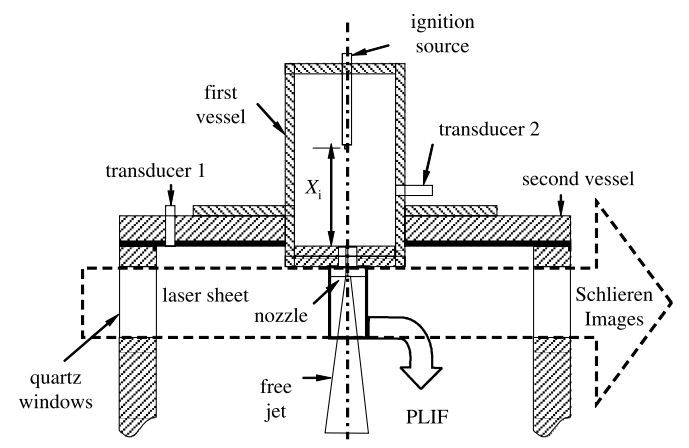

Fig. 1. Schematic of the combustion chamber.

distance can be adjusted by moving the spark plug along its axial direction.

The combustion leads to a pressure increase in the first chamber. The hot burned gases therefore expand through the nozzle into the second chamber, rendering a jet of hot burned gases that impinges into the unburned gas, possibly causing ignition. For this process, the ignition distance has an important effect: Reducing the ignition distance causes the resulting flame in the first chamber to reach the nozzle earlier. Therefore, a smaller fraction of the gas in the first chamber is burned at the time at which the flame enters the nozzle, and the pressure difference between the first and second chamber is smaller. The development of the hot jet and the subsequent ignition processes is imaged perpendicularly to the flow using ICCD cameras. The pressure trace in both chambers is recorded by means of transducers (Kistler Model 6031).

Figure 2 is a schematic sketch of the optical set-up used for the experiment. As excitation source for $\mathrm{OH}-\mathrm{LIF}$ a multiple $\mathrm{Nd}$ :YAG laser cluster consisting of four standard flash-lamppumped Nd:YAG lasers, each operated in double

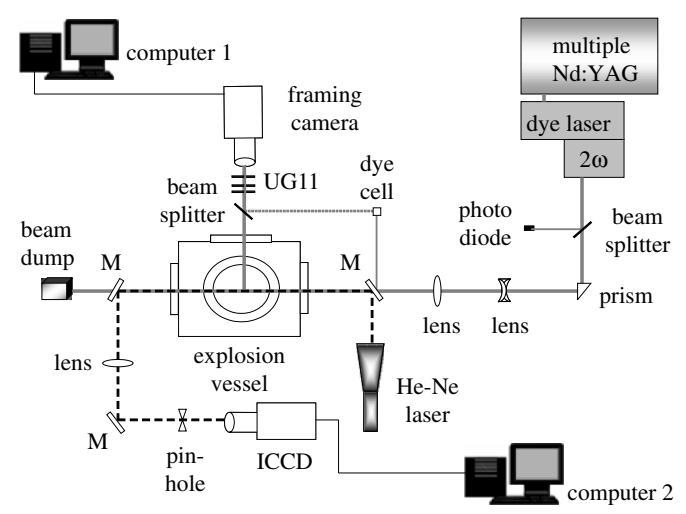

Fig. 2. Schematic of the optical set-up used for simultaneous OH-PLIF and Schlieren imaging. 
pulse mode with a variable pulse interval of 25-145 $\mu$ s was used. The beams from the four lasers were superimposed to a common optical axis and frequency doubled to $532 \mathrm{~nm}$. By interleaving the double pulses of the four lasers, the time delay between the pulses could be reduced down to $6.25 \mu \mathrm{s}$ in an eight-pulse sequence. The laser pulses for OH-PLIF were obtained by pumping a dye laser (operating on Rhodamine 590 as laser dye) with the Nd:YAG laser cluster and frequency doubling the dye laser output. For exciting the $\mathrm{OH}$ radical the dye laser was tuned to the $Q_{1}(9)$ transition near $283 \mathrm{~nm}$ in the $v^{\prime \prime}=0, \quad v^{\prime}=1 \quad$ vibrational band of the $\mathrm{A}^{2} \Sigma^{+}-\mathrm{X}^{2} \Pi$ electronic system of $\mathrm{OH}$. By means of cylindrical lenses and mirrors the laser beam was formed into a vertical sheet of approximately $39 \mathrm{~mm}$ in height and $200 \mu \mathrm{m}$ in thickness, and guided into the optically accessible test chamber described below. Subsequent fluorescence from the $\mathrm{OH}$ radical was observed using a combination of a high reflection filter (HR 275-295 nm) and a UG11 filter in front of the camera lens which eliminates reflections and scattering of the laser wavelength. A small part of the incoming laser sheet was reflected by a beam splitter and directed to a fluorescent dye cell. The resulting fluorescence from this dye cell was reflected onto the side of the same CCD as used for the $\mathrm{OH}$-LIF detection to enable correction for laser profile inhomogeneities. A small part of the output beam from the dye laser was guided onto a photodiode by means of a beam splitter. This allowed additional on-line monitoring of the laser pulse energies on a shot-to-shot basis.

The detector system used for the visualisation of planar OH-LIF is an ultra fast CCD camera (Imacon 468 , dynamic range 8 bit, $576 \times 385$ pixels). High framing speed is achieved by sequential exposure of eight individual CCD detectors with short exposure times. The CCD signals are digitised to 8 bits, and transferred to the controlling computer. Exposure times, gains and trigger delays are individually programmable for each $\mathrm{CCD}$, providing full timing control. More details of the laser cluster and the camera system can be found in [15].
The laser Schlieren optical system consists of a $\mathrm{He}-\mathrm{Ne}$ laser $(20 \mathrm{~mW}$ at $632.8 \mathrm{~nm})$ as a point source and a beam expander for collimating the beam. The beam is passed through the chamber along the same optical path as the dye laser sheet by means of mirrors. It is then redirected to a lens, which focuses the beam onto a dot mask where the Schlieren image is formed because of the light deflection due to the refractive index gradient inside the probe volume. For the laser Schlieren imaging, a Streak Star high-speed ICCD camera (La-Vision, 14-bit dynamic range, $384 \times 576-$ pixel array), coupled to a Sigma zoom lens (75-300, 4-5.6 f) was used. During each experiment four sequential Schlieren images with an exposure time of $1 \mu \mathrm{s}$ were recorded simultaneously with the OH-LIF images. The timing between the laser pulses and the different camera gate openings are adjusted by means of a delay generator circuit.

\section{Results and discussions}

A change in the ignition distance $X_{\mathrm{i}}$ leads to a change in the pressure ratio $p_{1} / p_{2}$ over the nozzle as the jet emanates out of the nozzle. Table 1 summarises the different nozzle diameters $d$ tested for each ignition distance $X_{\mathrm{i}}$ and the resulting pressure ratios over the nozzle. For a given ignition distance no fluctuations of the measured pressure ratio could be detected i.e., the uncertainty of this value is only determined by the inaccuracy of the pressure measuring system. Variations in ignition distances and nozzle diameters lead to a subsequent change in jet velocities and rate of cooling of the burned gas.

Experimental laser Schlieren and OH-LIF images for $p_{1} / p_{2}=2.21$ and $d=0.8 \mathrm{~mm}$ are shown in Figs. 3a and b, respectively. The boundary contours of the corresponding Schlieren images are drawn into the OH-LIF images for better comparison of the Schlieren- and LIF-images. The spatial and temporal evolution of the hot burned gas as it expands into the second chamber from the nozzle exit can be seen in the Schlieren sequences (Fig. 3a). The imaged area covers $16 \times 24.5 \mathrm{~mm}$. The first frame is taken $2678 \mu \mathrm{s}$

Table 1

Measurement parameters

\begin{tabular}{lllr}
\hline Ignition distance $X_{\mathrm{i}}(\mathrm{mm})$ & Nozzle diameter $d(\mathrm{~mm})$ & Pressure ratio $p_{1} / p_{2}$ & Ignition probability $(\%)$ \\
\hline 24 & 0.7 & 1.50 & 0 \\
24 & 0.8 & 1.44 & 61 \\
24 & 0.9 & 1.44 & 100 \\
32 & 0.8 & 2.21 & 15 \\
32 & 0.9 & 2.19 & 67 \\
32 & 1.0 & 2.06 & 100 \\
56 & 0.8 & 5.22 & 0 \\
56 & 1.1 & 4.75 & 89 \\
56 & 1.3 & 4.63 & 100 \\
\hline
\end{tabular}


$t=2678 \mu \mathrm{s}$
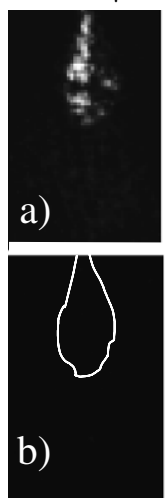

$t=2578 \mu \mathrm{s}$
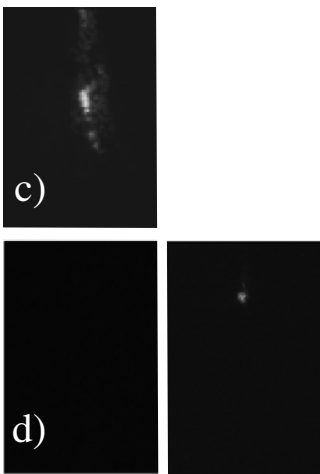

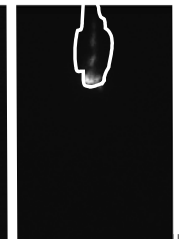

$t=2758 \mu \mathrm{s}$
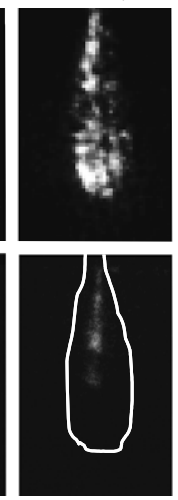

$t=2618 \mu \mathrm{s}$
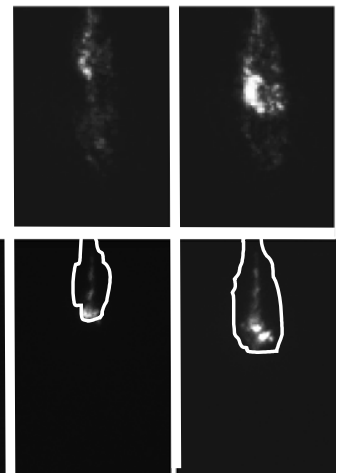

Fig. 3. (a) Simultaneous laser Schlieren and (b) OH-PLIF sequences, for $p_{1} / p_{2}=2.21$ and $d=0.8 \mathrm{~mm}$. The height of imaged area in (a) and (b) is $24.5 \mathrm{~mm}$. (c) Simultaneous laser Schlieren and (d) OH-PLIF sequences for $p_{1} / p_{2}=2.06$ and $d=1.0 \mathrm{~mm}$. The height of the imaged area is $39 \mathrm{~mm}$ in (c) and (d). The PLIF images have been recorded approximately $2 \mu$ s after the corresponding Schlieren images. The extra OH-LIF image (2nd from the left) in (d) has no corresponding simultaneous Schlieren image; it is shown here because it contains the first evidence of ignition in the sequence.

after the spark in the first chamber was triggered, and the period between each frame is $40 \mu \mathrm{s}$. Although LIF images were taken with a time delay of $20 \mu \mathrm{s}$ in the experiments, Fig. 3b shows only the time-resolved and simultaneous $\mathrm{OH}-$ LIF images corresponding to the Schlieren images of Fig. 3a recorded approximately $2 \mu$ s after the corresponding Schlieren images. From the recorded pressure trace and the OH-LIF images it can be inferred that there is no ignition in the second chamber in this case. Although some OH-LIF along the axis of the jet is visible, the absence of a significant amount of $\mathrm{OH}$ radicals at the nozzle exit indicates quenching caused by cooling due to the walls inside the nozzle before the gas expands into the second chamber. The structure that is visible in these Schlieren images is not associated with ignition and combustion, but simply to the mixing of the hot jet with unburned mixture.
Schlieren and OH-PLIF sequences for ignition of combustible gas by hot burned gas are shown in Figs. 3c and d, respectively, where the imaged area covers $26.5 \times 39 \mathrm{~mm}$. The images are shown for $p_{1} / p_{2}=2.06$ and $d=1.0 \mathrm{~mm}$. The first Schlieren image on Fig. $3 \mathrm{c}$ is captured $2578 \mu$ s after ignition in the first chamber. The time interval between the three different Schlieren frames is $40 \mu$ s and the time interval between the OH-LIF images is also $20 \mu$ s for the first, second and third LIF images and $40 \mu$ s between third and fourth LIF image. The PLIF images have been recorded approximately $2 \mu$ s after the corresponding Schlieren images. The region where ignition and subsequent combustion occurs can be clearly recognized by the sudden increase in OH-LIF intensity at $t=2598 \mu$ s (second OH-LIF image from the left; no corresponding Schlieren image is available for this point in time) at a distance of approximately $9 \mathrm{~mm}$ from the nozzle. The detection limit of the camera system is one critical factor with respect to the diagnostic analysis. However, the increase in $\mathrm{OH}$ concentration by several orders of magnitude during the ignition process helps in examining this process with reasonable accuracy using OH-LIF.

The interpretation of the LIF-signals is not straightforward, because the LIF-signal strength depends in a complicated way on several quantities (temperature, OH-number density, number density of species which cause quenching of the LIF-signal), and these quantities vary in a wide range in the system. It is not a priori clear if and how certain important processes in the jet (like ignition, combustion, and extinction) can be identified by the LIF-signal that they cause. As an aid to better interpret the image sequences, numerical simulations with a simple mixing reactor model [3] coupled with spectroscopic simulations [16] were performed. These simulations help in linking the observed signals (OH-LIF images) with the thermochemical state in the jet. The hot jet is represented in the simulations by a homogeneous constant-pressure reactor which is filled with hot exhaust gas with a certain initial temperature $T^{\text {(b) }}$ and initial chemical composition. To this gas, a flow of unburned hydrogen/air mixture $(T=300 \mathrm{~K}, p=1$ bar $)$ is added with a certain mixing rate $\theta$. Due to the addition of combustible fuel/air mixture to the hot jet, chemical reactions occur simultaneously to the mixing. The chemical source terms are calculated according to the set of elementary reactions for hydrogen/air combustion including 38 reactions of nine species [3]. The homogeneous reactor model allows large parametric studies concerning mixing rate and initial temperature. This enables us to link the $\mathrm{OH}$-signal to certain characteristic states that are associated with important processes inside the jet.

The model simulations yield the mixture composition and temperature for given inlet 
conditions as a function of time. From this information, the LIF-signal of the hydroxyl-radical $(\mathrm{OH})$ that results after excitation with a laserpulse of given spectral features and with a given detection bandpass was calculated. To this end, the program LASKIN [16] was used to compute the temporal evolution of the $\mathrm{OH}$ fluorescence intensity as a function of temperature, pressure, and chemical composition. The LIF-signal computation covers effects of the temperature dependent population of the laser-coupled ground state, line broadening of absorption lines by molecular collisions and by the Doppler-effect, as well as vibrational and rotational energy transfer from the laser-excited upper levels, and collisional quenching.

The initial composition is set to chemical equilibrium at a predefined temperature. The degree of mixing between hot gas jet and unburned gas is described by the mixture fraction $\xi$, defined as the fractional part of mass that stems from the burned gas. In the real jet, the mixing rate $\theta$ is not constant with respect to time. To mimic the decay of the mixing frequency with decaying shear velocity in the jet, a temporal decline of $\theta$ with decreasing mixture fraction $\xi$ is used in the model, according to $\theta=\theta_{0} \cdot \xi$, where $\theta_{0}$ is the mixing rate at $\xi=1$.

Figure 4a shows the temperature of the perfectly stirred reactor as a function of mixture fraction $\xi$ for different initial mixing rates $\theta_{0}$. The corresponding computed LIF-signals are shown in Fig. 4b. As can be seen in Figs. $4 a$ and $b$ for a fixed initial jet temperature $\left(T^{(\mathrm{b})}=1487 \mathrm{~K}\right)$, different mixing rates lead to qualitatively different behaviour: If the mixing rate is much greater than the chemical rate $\left(\theta_{0}=2 \times 10^{4} \mathrm{~s}^{-1}\right)$, chemistry can not compensate for the rapid cooling, and the system moves along the lower branch of Fig. 4a, namely the line of unreacted mixing states between unburned gas and the jet gas for different mixture fractions. The system will display virtually no OH-LIF signal in this case. The situation depicted in Figs. $3 \mathrm{a}$ and $\mathrm{b}$ is best represented by this "pure mixing branch" shown in Fig. 4.

If the chemical time scale is much smaller than the mixing time scale as represented by an initial mixing rate of $\theta_{0}=1 \mathrm{~s}^{-1}$ in Fig. $4 \mathrm{a}$, the unburned mixture is consumed instantaneously during mixing, leading to an increase in temperature. Correspondingly, there is an increase in $\mathrm{OH}$ number density leading to an increase in OH-LIF signal as seen in Fig. 4b. After complete mixing has occurred $(\xi=0)$, the system attains the adiabatic flame temperature corresponding to the unburned mixture. For intermediate mixing rates (as represented by the curves for $\theta_{0}=2 \times 10^{3} \mathrm{~s}^{-1}, \ldots$, $1.2 \times 10^{4} \mathrm{~s}^{-1}$ in Fig. 4), the system first follows the unreacted mixing line, but eventually, the temperature rise due to exothermic reactions outbalances the temperature decrease due to the addition of cold gas, and the system again ignites, combusts and finally evolves into chemical equilibrium. The OH-LIF signal intensity in this case displays a steep rise with a pronounced "overshoot", which is associated with a steep rise in temperature. Although the LIF-measurements do not supply quantitative information about $\mathrm{OH}$, the simple model calculations allow to link certain regions in the observed LIF-images with different states of the ignition/combustion process. In the measurements, only extremely weak or no LIF signals were observed in the jet directly at the nozzle exit. According to LIF-signal simulations for chemical equilibrium at different temperatures, the jet would emit LIF-signal that is
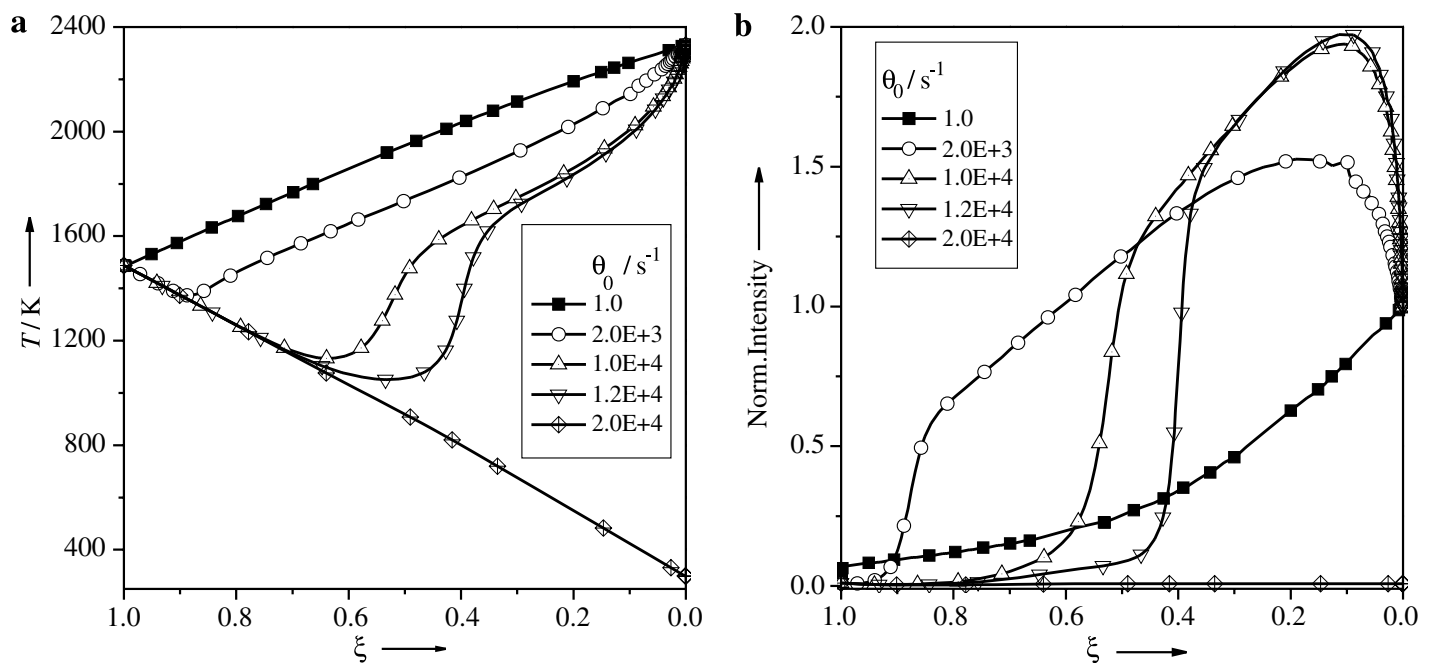

Fig. 4. Dynamic behaviour of the perfectly stirred reactor for $T^{(\mathrm{b})}=1487 \mathrm{~K}$ with different initial mixing rates $\theta_{0}$. 
detectable with the experimental setup, if its temperature was in the region around $1800 \mathrm{~K}$ or higher. It is therefore concluded that the heat loss of the jet to the nozzle walls is considerable, and that the jet temperatures in the experiments must be below $1800 \mathrm{~K}$ (compared to approximately $2390 \mathrm{~K}$ for chemical equilibrium without heat loss).

The ignition process is strongly influenced by temperature and mixing rate. In Fig. 5, the maximum mixing rate $\theta_{0, \max }$ at which ignition still occurs, is shown as a function of the exhaust gas temperature $T^{(\mathrm{b})}$. The curve separates the initial values of the mixing rate for a given temperature that lead to ignition from the initial values that do not lead to ignition. This temperature dependence is based on the dominant effect of the temperature on the elementary reactions. As the temperature $T^{(\mathrm{b})}$ increases, the induction time, which describes the time between the beginning of mixing and ignition, decreases. A high jet temperature is therefore required to ignite the mixture in cases of large mixing rates to overcome the cooling due to mixing.

The calculations can be used to explain some interesting experimental results. In Table 1, the dependency of the minimum nozzle diameter required for $100 \%$ ignition with pressure ratio is shown. The different ignition probabilities are based on at least 10 experiments per configuration. For example, for a pressure ratio of 1.44 the minimum nozzle diameter required for $100 \%$ ignition of combustible gases by hot burned gases is $0.9 \mathrm{~mm}$ while for $p_{1} / p_{2}=2.06$ it is $1.0 \mathrm{~mm}$. A higher outflow velocity resulting from the higher pressure difference leads to a higher mixing rate. Therefore, following Fig. 5, the nozzle diameter leading to $100 \%$ ignition has to increase with increasing pressure difference to decrease outflow velocity and increase temperature of the hot jet.

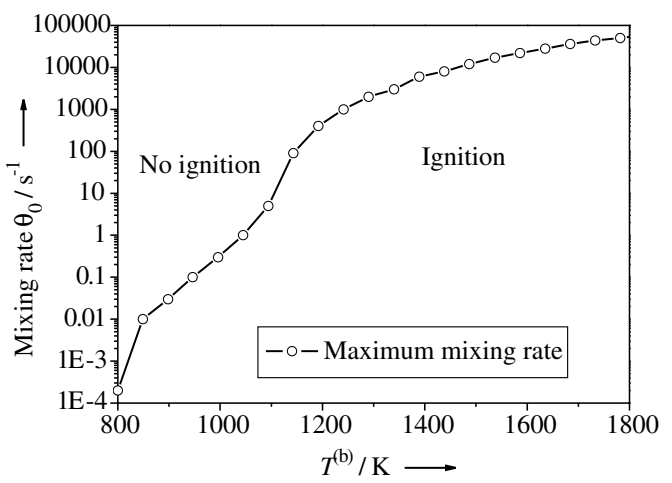

Fig. 5. Calculated (based on the mixing reactor model) maximum initial mixing rates still leading to ignition of unburned $28 \% \mathrm{H}_{2} / 72 \%$ air mixture as a function of the exhaust gas temperature $T^{(\mathrm{b})}$.
This effect of the increase in pressure ratio on the ignition behaviour can be seen in the Schlieren and PLIF images on Fig. $6\left(p_{1} / p_{2}=4.75\right.$ and $d=1.1 \mathrm{~mm}$ ). The imaged area starts at a distance of $29 \mathrm{~mm}$ from the nozzle exit and covers $26.5 \times 39 \mathrm{~mm}$. The time difference between the different Schlieren frames in Fig. $6 \mathrm{a}$ is $40 \mu \mathrm{s}$, and the first frame is recorded $3458 \mu$ s after ignition in the first chamber. The PLIF images in Fig. $6 \mathrm{~b}$ have been recorded approximately $2 \mu$ s after the corresponding Schlieren images. The initiation of the ignition of the gases by the hot jet happens in the time interval between the first and the second Schlieren frames, as is seen in the second frame of the PLIF sequence. Compared to the situation depicted in Figs. 3c and d, the ignition inside the free jet flow starts far more away from the nozzle exit at approximately $43 \mathrm{~mm}$. This increase of ignition distance from the nozzle exit inside the jet is mainly based on the higher pressure difference leading to a higher outflow velocity and mixing rate. Therefore following Figs. 4 and 5 the conditions are not favourable for ignition at the beginning and only mixing and cooling occurs. With increasing distance from the nozzle outlet, velocity and mixing decreases and finally at the experimental conditions in Fig. 6 the chemical reactions lead to an ignition, which again starts near the jet axis rather than at the sides. The localised ignition and extinction of kernels and the growth of the kernel is influenced by turbulent eddies, and the regions where OH-LIF signal is emitted are not spatially coherent. Note that there are also spot-like regions of considerably enhanced OH-LIF emission visible in Fig. 6. These bright spots can be understood using the results from the simple numerical model: They are regions that have just ignited and that are

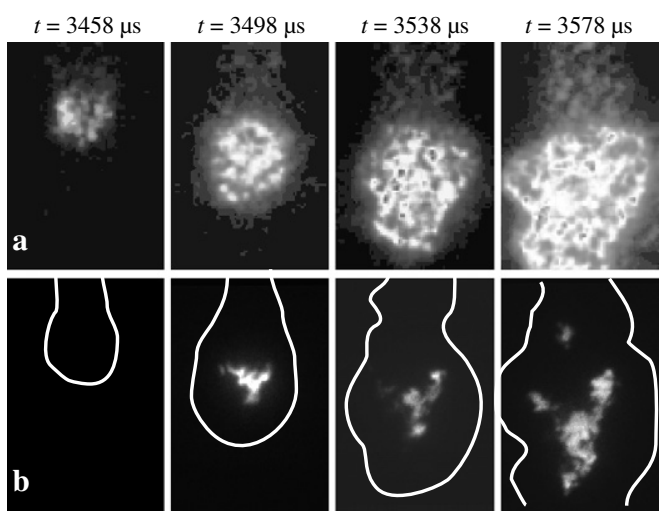

Fig. 6. (a) Simultaneous laser Schlieren and (b) OHPLIF sequences for $p_{1} / p_{2}=4.75$ and $d=1.1 \mathrm{~mm}$. The images are $39 \mathrm{~mm}$ in height, the top is located $29 \mathrm{~mm}$ away from nozzle exit. The PLIF images have been recorded approximately $2 \mu$ s after the corresponding Schlieren images. 
now on the branch of high OH-LIF emission ("overshoot"), not yet having reached chemical equilibrium. In Fig. 6, regions where ignition already has occurred and combustion takes place are visible, simultaneously with regions that are just igniting. Both the combusting and the igniting regions are not spatially coherent due to the influence of the turbulent flow caused by the jet.

The OH-signal always appears well inside the boundary of the Schlieren images (drawn as white lines into the OH-LIF images in Figs. 3 and 6). This does not per se mean that the $\mathrm{OH}$-signal source (as indicated by the occurrence of $\mathrm{OH}$ LIF) is located well inside the jet (rather than close to the boundary): the Schlieren images contain line-of-sight information of a three-dimensional object, projected onto the image plane, while the LIF images genuinely contain information from a two-dimensional region (the plane of the laser-sheet). The Schlieren boundaries plotted into the LIF-images therefore yield only rough information about the boundaries of the Schlieren region in the plane of the LIF-measurement. However, in all Schlieren/LIF picture pairs that were recorded it was seen that the region of ignition (indicated by the presence of enhanced OH-LIF signal) lies well within the zone marked by Schlieren images. From the combined information from all these images, we conclude that the OH-LIF emission does not happen at the jet boundary, but well within the jet near the tip.

\section{Summary and conclusion}

This paper reports on studies on the ignition of near-stoichiometric hydrogen/air mixtures by hot exhaust gas jets. In an optically accessible combustion cell, hot jet ignition experiments were performed under well-defined conditions. High-speed sequences of planar OH-LIF and Schlieren images were recorded to study the ignition of unburned hydrogen/air mixture by a hot exhaust gas jet. From these high-speed image sequences, the temporal development of individual ignition events could be tracked. To aid the interpretation of the observed LIF-signals, a simple numerical simulation of the reactive mixing of exhaust gas with $\mathrm{H}_{2}$ /air mixture was combined with spectroscopic simulations of the OH-LIF signal.

The Schlieren images show that the changes in pressure ratio across the nozzle substantially change the average outflow jet velocity. The absence of appreciable amount of $\mathrm{OH}$ LIF-signal at the nozzle exit suggests that the gas at this location is relatively cool due to heat losses to the nozzle walls. Subsequent ignition of the combustible mixture by the hot exhaust jets can be observed in the OH-PLIF sequences. The combination of mixing reactor model/spectroscopic simulations is used to link the observed LIF-signals with certain states (extinction, ignition, and combustion) of the hot jet/ $\mathrm{H}_{2}$-air mixing process. Especially, the interaction of turbulence and chemical reactions dominates the problem of hot jet ignition. This becomes apparent considering the locations in the jet where ignition is observed: The hot jet emerges from the nozzle with high velocity and underlies high shear stresses (strain rates) due to the contact with the surrounding $\mathrm{H}_{2}$ /air mixture. The observation that ignition occurs near the jet tip and not at the lateral sides of the jet can be explained with the different mixing behaviour of these areas. At the lateral sides the high shear stresses result in strong mixing, while at the tip mixing is reduced [17], increasing the probability of ignition in this area. The experimental data will be used as a basis for developing and validating detailed models of the hot jet ignition problem. Understanding this problem helps to improve the design of electrical equipment of the type of protection flameproof enclosure. Therefore, current experimental efforts are directed at determining instantaneous mixture fraction and temperature fields.

\section{Acknowledgments}

This work was supported by a grant under EU TMR program 'Access to Large Scale Facilities', Contract No. ERBFMGECT950020 (DG12), which is gratefully acknowledged. Also the Swedish Energy Agency and the Swedish Research Council are gratefully acknowledged for their support.

\section{References}

[1] E. Murase, S. Ono, K. Hanada, A.K. Oppenheim, Combust. Sci. Technol. 113 (1996) 167-177.

[2] IEC 60079-1, Electrical Apparatus for Explosive Gas Atmospheres-Part 1: Flameproof Enclosures ' $d$ ', 2003.

[3] U. Maas, J. Warnatz, Combust. Flame 74(1988) 53-69.

[4] J.D. Blouch, C.K. Law, Combust. Flame 132 (2003) $512-522$.

[5] H.G. Im, J.H. Chen, C.K. Law, Proc. Combust. Inst. 27 (1998) 1047-1056.

[6] J.W. Meyer, P.A. Urtiew, A.K. Oppenheim, Combust. Flame 14 (1970) 13-20.

[7] H. Phillips, Combust. Flame 19 (1972) 187-195.

[8] Ø. Larsen, R.K. Eckhoff, J. Loss Prev. Process Ind. 13 (2000) 341-347.

[9] M. Beyer, Ph.D. thesis, University of Braunschweig, 1997.

[10] T. Redecker, Classification of Flammable Gases and Vapours by the Flameproof Safe Gap and the Incendivity of Electrical Sparks. Report W-18, Physikalisch-Technische Bundesanstalt, 1981.

[11] IEC 60079-0, Electrical Apparatus for Explosive Gas Atmospheres-Part 0: General Requirements, 2004. 
[12] J. Warnatz, U. Maas, R.W. Dibble, Combustion, third ed., Springer, Berlin, 2001.

[13] A. Dreizler, S. Lindenmaier, U. Maas, J. Hult, M. Aldén, C.F. Kaminski, Appl. Phys. B70 (2000) 287-294.

[14] A.C. Eckbreth, Laser Diagnostics for Combustion Temperature and Species, second ed., Gordon and Breach, UK, 1996.

\section{Comments}

Katharina Kohse-Höinghaus, Universität Bielefeld, Germany. Your results from the modeling seem to indicate that in some intermediate cases (between the limits of no ignition/no OH vs. complete ignition/full $\mathrm{OH}$ signal) there may be situations where the expected signal will not unambiguously depend on the conditions of the gas mixture. Please comment on the potential predictive capability of ignition under these intermediate conditions.

Reply. We do not attempt a complete identification of the conditions in the gas mixture (temperature, mole fractions of all species). What we do obtain from the model simulations is information about how certain characteristic processes in the system (like extinction, or ignition/combustion at high mixing rates) show up in the OH LIF images.

Mohy S. Mansour, NILES-Cairo University, Egypt. The boundary conditions of the hot exhaust gases are not well defined due to the shot-to-shot variations. So, validation of the numerical calculations based on the present experimental data is not accurate enough. A well defined source of hot exhaust gases, providing definite boundary conditions, should improve the validation
[15] J. Hult, M. Richter, J. Nygren, M. Aldén, A. Hultqvist, M. Christensen, B. Johansson, Appl. Opt. 41 (2002) 5002-5014.

[16] U. Rahmann, A. Bülter, U. Lenhard, R. Düsing, D. Markus, A. Brockhinke, K. Kohse-Höinghaus, Faculty of Chemistry, Physical Chemistry I, 2003, available at $<$ http://pc1.uni-bielefeld.de/ laskin/ $>$.

[17] G. Bruneaux, SAE technical paper 02-SFL39, 2002.

and interpretation of the present data. Can you comment on this?

Reply. As mentioned in the paper, the experiments are not used here to validate numerical calculations. Instead, the numerical simulations are used to aid the interpretation of the experimental results, namely the $\mathrm{OH}$ LIF-signals. The connection of the LIF-signal to processes like ignition or extinction is essentially the same for a wide range of boundary conditions (e.g., initial jet temperatures). For our study, therefore, uncertainty in boundary conditions was not an issue.

G.I. Nathan, University of Adelaide, Australia. You explained your result in terms of mixing. Did you consider the effect of strain? The mean scalar field is not a reliable measure of molecular mixing.

Reply. The effect of strain was not included in the numerical simulations presented here. We have performed similar studies using unsteady 1-D flame simulations, which do include strain effects. They deliver similar connections between OH-LIF signal and the important processes like ignition or extinction. 